\title{
Einfluss der englischen Sprache auf die deutsche Sprache
}

\author{
إعـــداد \\ اشرف سمير محمد أحمد \\ مدرس بكلية الألسن - جامعة بنى سويف مجمي
}

مجلة الاراسات التربوية والانسانية ـ كلية التربية ـ جامعة دمنهور

المجلدالحادى عشر - العدد الثالث - لسنة 2019 
مجلة الدراسات التربوية والانسانية ـ كلية التربية ـ جامعة دمنهور ـ المجلد الحادى عثر - العدد الثانى - لسنة 9 1 ـ r

\section{Einfluss der englischen Sprache auf die deutsche Sprache}

\section{Einleitung}

Im Zuge der Globalisierung ist Englisch in größeren Teilen der Welt zu einer Lingua Franca avanciert. Der öffentliche Diskurs über den Einfluss des Englischen auf die deutsche Sprache vernachlässigt jedoch eine lange und enge Geschichte zwischen den beiden Sprachregionen, in der regelmäßig sprachliche Übertragungen stattgefunden haben. Folglich sind Behauptungen über die mögliche Gefährdung des Deutschen keineswegs ein Phänomen des 20. Jahrhunderts, sondern ein natürlicher Teil des Sprachkontakts im Laufe der Zeit.

Da wurde die Übertragung von englischen Krediten durch die neuen Medien erleichtert, und ihre Integration ist nicht mehr eine Frage vergangener Zeiten, sondern kann und muss von den Sprechern der modernen deutschen Sprache ausgeführt werden, die Art und Weise, wie die Anglizismen behandelt werden, scheint sich zu verändern. Nicht nur Einstellungsfaktoren für den Gebrauch von Anglizismen, sondern auch deren Integration in das deutsche morpho-syntaktische System könnten beeinflussen, wie sich die deutsche Sprache unter dem zunehmenden Einfluss des Englischen entwickelt.

In diesem Sinne werde ich versuchen, die Kontaktsituation zwischen den beiden Sprachen Englisch und Deutsch wie in der Vergangenheit und wie sie sich bis heute entwickelt hat, zu beschreiben. Auf der Grundlage einer Untersuchung, wie Anglizismen in das deutsche morpho-syntaktische System integriert werden, hoffe ich, Vorhersagen über die zukünftige Entwicklung des Kontaktszenarios und seine Einflüsse für das Deutsche treffen zu können.

Außer Acht lässt, dass deutsche Wörter auch ins Englische übertragen werden sind sowie strukturelle Anglizismen und Hybridverbindungen im Deutschen. Obwohl diese Übertragungen stattfinden, wird es keinen Raum geben, sie ausführlich $\mathrm{zu}$ diskutieren. Stattdessen konzentriert sich diese Forschung ausschließlich auf lexikalische Übertragungen und Pseudo-Kredite und versucht, die Veränderungen, die sie in das moderne Deutsche bringen, nachzuvollziehen.

\section{Die Kontaktsituation zwischen Englisch und Deutsch}

Englisch kann selbst als westgermanische Sprache eingestuft werden, was die lexikalischen Übertragungen zwischen Englisch und Deutsch in zweierlei Hinsicht erheblich erleichtert hat: Die beiden Sprachen ,sind r.o 
in ihrer historischen Entwicklung sehr ähnlich und teilen eine hohe lexikalische Affinität ${ }^{\text {"1 }}$. Der wesentliche linguistische Unterschied liegt in der Entwicklung ihres Flexionssystems im Laufe der Zeit: „In Bezug auf ihre morphologischen Strukturen hat Englisch jedoch fast alle seine germanischen Beugungen verloren und ist zum analytischen Ende des Spektrums hin verschoben, wohingegen Deutsch seine Flexionskategorien und seine synthetische Natur hauptsächlich beibehalten hat. ${ }^{\text {"2 }}$ Infolgedessen hat Deutsch Beugungen für Verben (Person, Spannung, Zahl und Stimmung) sowie für Substantive (Fall, Zahl, Geschlecht) behalten; ein Paradigma, das Schwierigkeiten bei der Integration von Lehnwörtern aufzeigt.

\subsection{Sprachkontakt - Eine Einführung}

Wenn zwei oder mehr Sprachen miteinander in Kontakt kommen, kann das Ergebnis ihrer Interaktion in einer Reihe von linguistischen Phänomenen beobachtet werden. Wie Thomason feststellt, kann der Einfluss je nach Intensität der betreffenden Sprachen zu direkten Importen aus einer Ausgangssprache führen, entweder allein durch Morphemübertragung, Morphem- und Strukturübertragung oder allein durch Übertragung der Struktur; und diese Merkmale der Ausgangssprache können während des Übertragungsprozesses strukturell modifiziert werden oder nicht $^{3}$. In Kontaktszenarien mit geringer Intensität werden in der Regel nur nicht-basische Wörter ausgeliehen, während mehr Elemente einer Sprache mit steigender Kontaktintensität zu Veränderungen neigen, so dass allgemein davon ausgegangen wird, dass Vokabular vor Struktur geliehen ist ${ }^{4}$. Als allgemeine Tendenz bestätigt Onysko, dass Kontakt auf der lexikalischen Ebene der Sprache am ehesten wahrnehmbar ist, während systemische, d.h. grammatische Muster eine größere Stabilität für kontaktbedingte Veränderungen zeigen ${ }^{5}$.

${ }^{1}$ Vgl. Onysko, Alexander: Anglicisms in German, 2007, S. 49.

2 Vgl. Onysko, Alexander: Anglicisms in German, 2007, S. 49.

${ }^{3}$ Vgl. Thomason, Sarah G.:Language Contact, 2001, S. 69

${ }^{4}$ Ebda. S. 62

${ }^{5}$ Vgl. Onysko, Alexander: Divergence with a Cause? The Systematic Integration of Anglicisms in

German as an Indication of the Intensity of Language Contact; 2009, S. 55.

\section{DOI: 10.12816/0054838}


Da der Sprachkontakt jedoch ein zutiefst sensibles Forschungsfeld ist, hat sich bis auf wenige Ausnahmen fast jede Beschränkung auf die Art und Weise der Ausleihe von Sprachmerkmalen als falsch erwiesen ${ }^{6}$

Vor allem weil Sprachkontakt das Ergebnis von Menschen in Kontakt ist und von Gemeinschaften von Menschen mit unterschiedlichen sprachlichen Hintergründen in Kontakt sind ${ }^{7}$, können keine definitiven Verallgemeinerungen für ein Forschungsgebiet gezogen werden, das vollständig auf Menschen und ihren persönlichen Einstellungen beruht. Wie Thomason erklärt, sind diese Einstellungen der Sprecher : der Joker in diesem Bereich; sie können und verursachen manchmal Verletzungen der meisten allgemein gültigen Vorhersagen über kontaktbedingte Veränderungen. ${ }^{8}$ Obwohl dadurch keine eindeutigen Regeln gebildet werden können, ist es dennoch möglich, zumindest Möglichkeiten und Wahrscheinlichkeiten für die Entwicklung von Kontaktsprachen zu formulieren. $\mathrm{Zu}$ den sozialen Prädiktoren für kontaktbedingte Veränderungen zählen neben der Einstellung der Sprecher auch die Dauer der Kontaktperiode, die Größe der Sprechergruppen und die streitbare sozioökonomische Dominanz ${ }^{9}$. Weitere potentiell wichtige Variablen sind aufgelistet. ${ }^{10}$

\subsection{Die Vergangenheit: Phasen der lexikalischen Übertragung zwischen Englisch und Deutsch}

Was die Übertragungen zwischen Englisch und Deutsch betrifft, lassen sich mehrere Phasen unterscheiden, in denen die Intensität zwischen den beiden Sprachen zu einem Anstieg der Lehnwörter in Deutsch führt. Die sprachliche Nähe ist das Ergebnis eines intensiven kulturellen Austauschs zwischen Deutschen und Briten: Sie stammt aus dem fünften Jahrhundert, als germanische Siedler, die England eroberten, aus dem heutigen Norddeutschland und Süddänemark kamen, und im achten Jahrhundert, als ein Großteil Deutschlands von Iren und Engländern christianisiert wurde ${ }^{11}$. Als germanische Siedler ihre germanische Sprache mitbrachten, um das bestehende Celticum zu ersetzen, war die Bühne für einen beständigen linguistischen Austausch geschaffen. Die folgenden Absätze versuchen, die Wellen der aus dem Englischen

\footnotetext{
${ }^{6}$ Vgl. Thomason: 2001, Kapitel 3.2.

${ }^{7}$ Vgl. Clyne, Michael : Dynamics of Language Contact: English and Immigrant Languages. Cambridge, 2003, S. 1.

${ }^{8}$ Vgl. Thomason, 2001, S. 61.

${ }^{9}$ Ebda: S. 66.

${ }^{10}$ Vgl. Aikhenvald, Alexandra Y: "Grammars in Contact.....", 2007, S. 55.

${ }^{11}$ Vgl. Görlach, Manfred: "Introduction." English in Europe..." , 2007, S. 13.

$r \cdot V$
} 
kommenden deutschen Sprache zu beleuchten und bestimmte Phasen zu skizzieren von intensivem Sprachkontakt mit besonderer Rücksicht auf geliehene Gegenstände, die noch heute häufig verwendet werden. Im Mittelalter war der Einfluss von Angelsächsisch und Englisch auf Deutsch extrem klein. Die wenigen englischen Lehnwörter im Spätmittelalter waren technische Ausdrücke aus der Welt der Seefahrt ${ }_{12}^{2}$, z.B: die vorliegenden Begriffe Boot und Lotse (von loetsman). Diese Anleihen waren selten und seit so langer Zeit in der Sprache, dass viele von ihnen heute assimiliert und für deutsche Muttersprachler fast unkenntlich sind ${ }^{13}$. Erst nach dieser Phase des relativ spärlichen Sprachkontakts im Mittelalter begann sich die Übertragung englischer Wörter ins Deutsche zu beschleunigen. Die Mitte des siebzehnten Jahrhunderts brachte häufige Anpassung der politischen Begriffe als England allmählich von politischem Interesse, vor allem nach der Hinrichtung von Charles I. im Jahr 1648 und die folgenden Berichte über die Ereignisse aus dem Englischen übersetzt ${ }^{14}$. Wie Viereck weiter bemerkt, war es daher nicht verwunderlich, dass englische Ausdrücke aus dem politischen Bereich in dieser Zeit entweder in ihrer ursprünglichen Form ins Deutsche gebracht oder ins Deutsche übersetzt wurden ${ }^{15}$, ein Beispiel ist Flutlicht. Die englische Wissenschaft spielte auch eine Rolle im Sprachkontakt des 17. Jahrhunderts und war eine konstante Quelle für Begriffe wie Logarithmus und Zentrifuge ${ }^{16}$.

Im 18. Jahrhundert kam es zu einem intensiven Kontakt zwischen Englisch und Deutsch, insbesondere auf intellektueller und kultureller Ebene. Als Konsequenz fanden große Teile des englischen Vokabulars ihren Weg ins Deutsche. ${ }^{17}$ Vor allem war der Einfluss der englischen Literatur, sozialer Praktiken, historischer und philosophischer Schriften

${ }^{12}$ Vgl.Viereck, Wolfgang : "The influence of English on German in the past and in the

Federal Republic of Germany.", 1986, S. 107.

${ }^{13}$ Vgl.Görlach, Manfred and Ulrich Busse: "German." English in Europe... "2007, S. 13.

${ }^{14} \mathrm{Vgl}$. Viereck, Wolfgang: "The influence of English on German in the past and in the Federal Republic of Germany.", 1986, S. 107.

${ }^{15}$ Ebda. S. 107.

${ }^{16}$ Ebda. S. 108.

${ }^{17}$ Ebda. S. 108.

DOI: $10.12816 / 0054838$ $r \cdot \Lambda$ 
und der kulturellen Auswirkungen in Architektur, Gartenbau, Töpferei etc. und in den Wissenschaften / Technologien auf dem Höhepunkt ${ }^{18}$.

Im neunzehnten Jahrhundert begann die Technologie. Da war der Wortschatz über den Aufstieg der industriellen Revolution und zwar: britischer technologischer Methoden in Schiffbau, Eisenbahntechnik, Bergbau, Stahlerzeugung, Weberei und Tuchmacherei wurde in weiten Teilen Europas allgemein akzeptiert. ${ }^{19}$ Es ist interessant $\mathrm{zu}$ bemerken, dass bei den meisten Krediten die Kreditaufnahme des Gegenstandes selbst die Einführung eines Wortes begleitete und damit die wirtschaftlichen Auswirkungen Englands auf den industriellen Bereich hervorhob. ${ }^{20}$

Görlach und Busse zeichnen eine weitere Differenzierung im Sprachkontakt des Jahrhunderts, als in seiner zweiten Hälfte : Wortschatz zu verschiedenen Sportarten (z. B. Fußball, Golf, Tennis und Pferderennen) sowie Bezeichnungen aus dem Alltagsleben wie Getränke, Kleidung, Hunderassen wurden aus England importiert und bereicherten den deutschen Wortschatz erheblich. ${ }^{21}$

Das zwanzigste Jahrhundert kann in drei diskrete Perioden der Kreditaufnahme aus dem Englischen unterteilt werden. Während zu Beginn des 20. Jahrhunderts zahlreiche AME-Begriffe (american music environments) aus den Bereichen Musik, Tanz, Automobil und Luftfahrt ins Deutsche gelangten, wurde die Wirkung nach 1945 massiv: Die kulturelle Ausrichtung auf die USA trieb die zunehmende Einführung anglo-amerikanischer Lehnwörter $\operatorname{voran}^{22}$. Es ist interessant festzustellen, dass vor allem Begriffe aus dieser Zeit ,sich einen sicheren Platz im deutschen Wortschatz erobert haben", wie Viereck betont. $^{23}$ Darunter befinden sich Begriffe, die einen Naturalisierungsprozess durchlaufen haben, der heute noch häufig verwendet wird und nicht mehr mit ihrer ursprünglichen englischen Etymologie vergleichbar ist. $\mathrm{Zu}$ diesen Wörtern gehören unter anderem: aus der ersten Phase, Untergrundbahn (aus engl. U-Bahn-Linie), Globetrotter, Thermosflasche, Polo, Golf, Bluff, und für die

${ }^{18}$ Vgl.Görlach, Manfred and Ulrich Busse: "German." English in Europe... "2007, S. 13.

${ }^{19}$ Ebda: S. 14.

${ }^{20}$ Vgl. Viereck: 1986, S. 109.

${ }^{21}$ Vgl. Görlach, Manfred and Ulrich Busse: "German." English in Europe... "2007, S. 14.

${ }^{22}$ Ebda. S. 14.

${ }^{23}$ Vgl. Viereck, Wolfgang : "The influence of English on German in the past and in the Federal Republic of Germany.", 1986, S. 111.

r. 9 
Zwischenkriegszeit, Badminton, Lautsprecher, Einbahnstraße, Wochenende und Sex Appeal. ${ }^{24}$ Die dritte Periode des 20. Jahrhunderts hat im Zuge der Globalisierung stattgefunden:

Seit den 1990er Jahren sind die Auswirkungen der amerikanischen Kultur und ihrer linguistischen Einflussreflexe stärker geworden. Die weltweite Kommunikation über das Internet, die Globalisierung der Volkswirtschaften, die zu multinationalen Konzernen geführt hat, und das kommerzielle Fernsehen mit seinen Werbeanzeigen und Videoclips haben zu einer neuen Dimension lexikalischer Anleihen und Code-Switching geführt, zumindest in den Fachsprachen Wirtschaft, Werbung und Jugendsprache. Dieser ungebremste Zustrom hat teilweise feindselige Reaktionen hervorgerufen. ${ }^{25}$

In der Summe haben kultureller und in geringerem Maße der geographische Kontakt dazu beigetragen, den Sprachkontakt zwischen Englisch und Deutsch zu erleichtern, um Kredite zu ermöglichen. Da Sprache Teil unserer Kultur ist, spiegelt ihr Kontakt wider, wie sich die Beziehungen zwischen Nationalitäten im Laufe der Zeit entwickelt haben. Insbesondere im Hinblick auf die Kreditaufnahme aus dem 19. und 20. Jahrhundert ist es offensichtlich, dass der enorme Zustrom an technischer Innovation in Deutschland eine angemessene Terminologie erforderte. Und was war einfacher, als den im Herkunftsland verwendeten Begriff zu borgen? Da das Label zusammen mit dem Begriff in die Sprache eindringt, hat manches Wort unabhängig von der Intensität des Kontakts in eine völlig andere Sprache Eingang gefunden. Englisch selbst hat sein Lexikon in großem Maße erweitert, indem es einfach ausländische Sprachen übernommen und sie neben dem englischen Äquivalent eingesetzt hat, was Differenzierungen zwischen verschiedenen Nuancen ermöglicht.

\subsection{Die Gegenwart: die Kontaktsituation zwischen Englisch und Deutsch heute}

Die oben erwähnten Phasen der lexikalischen Übertragung haben Deutsch mit einem beträchtlichen Teil des englischen Wortschatzes bereichert. Um dieses Bild zu vervollständigen, ist es notwendig, das Kontaktszenario zwischen diesen beiden Sprachen, wie

\footnotetext{
${ }^{24}$ Ebda. S. 111.

${ }^{25}$ Vgl. Görlach, Manfred and Ulrich Busse: "German." English in Europe... "2007, S. 14.
}

\section{DOI: 10.12816/0054838}


es heute ist, $\mathrm{zu}$ definieren und somit die Intensität der Auswirkungen, die Englisch auf das moderne Deutsch hat, zu bewerten.

Laut Onysko ist die Situation zwischen Englisch und Deutsch als ,,remote ${ }^{6} \mathrm{zu}$ bezeichnen, in der der Kontakt, nicht auf direkter Sprecherinteraktion beruht, sondern primär durch Print- und audiovisuelle Medien (Zeitungen, Zeitschriften, TV, Radio, Internet)

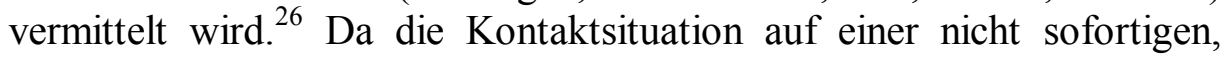
entfernten Beziehung beruht, werden nur abgeschwächte Einflüsse (wie lexikalische Kreditaufnahme) erwartet. $^{27}$

Obwohl dieses Kontaktszenario als fern eingestuft wird, lassen die verschiedenen Einflusstypen Rückschlüsse auf die Auswirkungen von Englisch auf Deutsch zu. In diesem speziellen Fall ist zu erwähnen, dass obwohl die struktur der englischen und deutschen Sprache wider erwarten nur eine geringe Anzahl von Direktkrediten und eine relativ geringe Anzahl von Calques möglich sind. Görlach und Busse zeigen somit, dass das heutige Deutsch im Vergleich zu anderen Sprachen im modernen Europa in höchstem Maße Anglizismen offen steht. ${ }^{28}$ Wie es Viereck nennt:

Die Anzahl der direkten und indirekten deutschen Kredite aus dem Englischen auf lexikalischer Ebene ist extrem hoch. Gerade die direkte Anleihe aus amerikanischem Englisch und britischem Englisch also die Null-Substitution - ist ein deutliches Zeichen der zunehmenden Technisierung und Internationalisierung in der deutschen Wortschöpfung von heute. $^{29}$

Der Gebrauch von Anglizismen im Deutschen bezieht sich daher nicht nur auf bestimmte Domänen, sondern auch auf unterschiedliche Einstellungsfunktionen. Wie Görlach und Busse angeben, sind die meisten Anglizismen im Deutschen entweder ,technisch“ (beschränkt auf die Terminologien von Naturwissenschaften, Technologien und anderen Jargons); diese sind selten, unvollständig integriert, geschrieben und verhaltensneutral ,oder" umgangssprachlich / umgangs-sprachlich; diese sind häufig nur in der Jugendsprache, im Journalismus und in der Werbung $\mathrm{zu}$ finden und typisch für den gesprochenen Gebrauch. Im

\footnotetext{
${ }^{26}$ Vgl. Onysko: 2009, S. 58.

${ }^{27}$ Ebda: S. 58.

${ }^{28}$ Ebda. 2007, S. 31.

${ }^{29}$ Vgl. Viereck: 1986, S. 119.

r)
} 
Gegensatz zu den Begriffen der [ersten Kategorie] ist die Bedeutung umgangssprachlicher Begriffe oft vage. ${ }^{30}$

Zusätzlich ist das Kontaktszenario zwischen Englisch und Deutsch nicht nur klassifiziert nach den verschiedenen Bereichen der Diskurs- und Einstellungsfunktionen,ihre Verwendung in bestimmten sozialen Umgebungen sollte berücksichtigt werden: Es liegt auf der Hand, dass der Einfluss von Englisch auf Deutsch im Wortschatz ungleich verteilt ist, was Domänen, Formalitäten, technische Aspekte, Häufigkeit und soziale Variablen (Alter, Bildung, Region) betrifft. ${ }^{3}$

Zusammenfassend lässt sich sagen, dass die hohe Zahl der Direktkredite statt der bloßen Cal- quing einen extremen Einfluss auf Deutsch vermuten lässt. Auf der anderen Seite Sprecher-externe soziokulturelle Faktoren (einschließlich Demographie und Diskursfelder), Sprecher-bewusste Einstellungsparameter (wie Fragen des Prestiges und Sprach-Purismus) und nicht zuletzt unbewusste linguistische Kriterien (z. B. eine zugrunde liegende Kredithierarchie) ) kann und wird beeinflussen, wie sich der Sprachkontakt zwischen Englisch und Deutsch entwickelt.

Im Nachhinein könnte die Tatsache, dass in bestimmten Sprachgebieten Anglizismen auftreten, einen größeren Einfluss auf die Gesellschaft haben als angenommen. Vor allem die Verwendung von Anglizismen im Alltagsdiskurs, etwa in Lifestyle - und Unterhaltungsdomains (und gerade diese Begriffe wurden im Deutschen so stark genutzt, dass sie semantisch leer werden) könnte Teile der Sprechergemeinschaft in größerem Umfang beeinflussen als ihre einzige in eingeschränkten Domänen. Massenmedien absorbieren leicht Anglizismen, und alltägliche Begriffe werden eingebürgert und in die Sprache integriert, mit der Konsequenz, dass viele englische Lehnwörter verwendet werden. Es ist daher notwendig, nach der Definition in welchen Kontexten Anglizismen verwendet werden, die Einstellungen zu ihrer Verwendung zu betrachten, um einen vollständigen Überblick über ihre Verwendung im heutigen Deutsch zu erhalten.

${ }^{30}$ Vgl. Görlach, Manfred and Ulrich Busse: "German." English in Europe... "2007, S. 28.

${ }^{31}$ Ebda: S. 28.

DOI: $10.12816 / 0054838$ 
مجلة الدراسات التربوية والانسانية ـ كلية التربية ـ جامعة دمنهور ـ المجلد الحادى عثر - العدد الثانى - لسنة 9 1 ـ r

\section{Anglizismen auf Deutsch}

\subsection{Definition}

Wie bei den meisten Definitionen bleiben die Versuche, zu definieren, was als Anglizismus gilt, höchst umstritten. Vor allem die restriktiven Grenzen dessen, was unter diese Definition fallen soll, sind verschwommen, und es erweist sich als schwierig, eine präzise und umfassende Definition zu finden. ${ }^{32}$

Vermutlich eine der umfassendsten und aktuellsten Definitionen wurde von Onysko im Jahr 2009 gemacht, als er seine erste Definition von 2007 erweiterte - was definiert, dass Anglizismus ein Beispiel für ein englisches lexikalisches, strukturelles und phonologisches Element im Deutschen ist, das formell mit Englisch verwandt werden kann. ${ }^{33}$ Es ist daher offensichtlich, dass Anglizismen nicht klar als lexikalischer oder anderweitig offensichtlicher Gegenstand umrissen werden können, sondern auch sprachimmanente Merkmale umfassen können, die viel schwieriger zu erkennen sind.

Görlach geht auf die Einflüsse auf die Morphologie, Syntax, den Stil und die Progamatik der aufnehmenden Sprachen aus, schließt sie jedoch von seinem Forschungsschwerpunkt aus. Zusammen mit Busse konzentriert sich Görlach auf unkomplizierte Kredite als die Art von Einfluss, der am leichtesten zu erkennen ist und der sich durch ihre Fremdschreibung, Aussprache und /oder Morphologie auszeichnet. ${ }^{34}$

Englische Wörter jedoch, die auf der Grundlage lateinischer oder griechischer Elemente geprägt sind und daher in ihrer deutschen Form z.B: Telegramm, Fernsehen oder Telefon, keine besonderen englischen Merkmale aufweisen, werden von Görlach und Busse als „Internationalismen“ bezeichnet und sind somit auch aus dem Wörterbuch des europäischen Anglismus ausgeschlossen. ${ }^{35}$

Die Frage, ob Kreditübersetzungen (oder Calques) in die Definition von Anglizismen aufgenommen werden sollten, ist unter Wissenschaftlern höchst fragwürdig. Ausgehend von der Annahme, dass Kredite, die ausschließlich aus deutschen Morphemen bestehen, nur schwer als englisch identifiziert werden können und sie keinen großen

${ }^{32}$ Vgl. Plümer, Nicole: Anglizismus - Purismus - Sprachliche Identität.

Frankfurt am Main: Peter Lang, 2000, S. 19.

${ }^{33}$ Vgl. Onysko: 2007, S. 90.

${ }^{34}$ Vgl. Görlach, Manfred and Ulrich Busse: "German." English in Europe... "2007, S. 15 .

${ }^{35}$ Ebda: S. 28.

YIT 
Anteil am Kreditprozess haben. ${ }^{36}$ Viele Forscher haben sie von ihrer Definition von Anglizismen ausgeschlossen. Wie Görlach und Busse zeigen, ist Kreuzworträtsel eine Übersetzung aus dem englischen crossword puzzle - aber seine Form ist ganz deutsch. ${ }^{37}$

Onysko erklärt den problematischen Stand der Kreditübersetzungen, wenn er argumentiert, dass die Kombination von translationaler Äquivalenz und struktureller Ähnlichkeit eines Begriffs in zwei Sprachen keinen lexikalischen Transfer beweist, wenn der Wortbildungsprozess in den fraglichen Sprachen produktiv ist. ${ }^{38}$ Darüber hinaus reicht die Koexistenz desselben Begriffs in zwei Sprachen nicht aus, um zu definieren, in welcher der Sprachen der Begriff zuerst entstand, eine Frage, die nur durch kulturhistorische Analysen beantwortet werden kann. ${ }^{39}$ Angesichts dieser Schwierigkeiten, die die Frage, ob Leihübersetzungen in die Definition von Anglizismen einbezogen werden sollen, sehr kontrovers erscheinen lassen, sollen sie aus meinen Analysen im Hinblick auf die weitere Lektüre ausgeschlossen werden.

Ein weiterer wichtiger Punkt ist der Prozess, durch den Anglizismen eintreten. Die deutsche Sprache ist keineswegs auf direkte Kredite in eine Richtung beschränkt, sondern kann das Ergebnis eines deutschen Neologismus sein: Während alle Anleihen als Anglizismen gelten, sind nicht alle Anglizismen tatsächlich das Ergebnis eines Kreditaufnahmeprozesses. ${ }^{40}$

Zusammenfassend ist es notwendig, die Prozesse zu untersuchen, mit denen englische Wörter in der deutschen Sprache verwendet wurden, um Verwechslungen mit Begriffen aus der deutschen Kreativität zu vermeiden. Dies gilt sowohl für Calques, die zugegebenermaßen schwieriger $\mathrm{zu}$ erkennen sind, als auch für das Phänomen der Pseudo-Anglizismen, auf die im Folgenden näher eingegangen wird.

\footnotetext{
${ }^{36}$ Vgl. Yang, Wenliang : Anglizismen im Deutschen. Tübingen: May Niemeyer Verlag., 1990, S. 15.

${ }^{37}$ Vgl.Görlach, Manfred and Ulrich Busse: "German." English in Europe... "2007, S. 15.

${ }^{38}$ Vgl. Onysko: 2007, S. 26.

${ }^{39}$ Ebda. S. 26.

${ }^{40}$ Vgl. Onysko, Alexander: Anglicisms in German: Borrowing, Lexical

Productivity, and

Written Codeswitching. Berlin, 2007, S. 11.
} 
مجلة الدراسات التربوية والانسانية ـ كلية التربية ـ جامعة دمنهور ـ المجلد الحادى عشر - العدد الثانى - لسنة 9 ـ ب ب

\subsection{Wörter Klassen von Anglizismen auf Deutsch}

Wie die Hierarchie der Kreditfähigkeit andeutet, werden Substantive häufiger ausgeliehen als andere, wenn zwei Sprachen miteinander in Verbindung stehen. Dies liegt vor allem daran, dass sie eine offene Wortklasse sind, die eine große Erweiterung des Wortschatzes ermöglicht. In der Folge sind es hauptsächlich Substantive, die im Deutschen unter Anglizismen zu finden sind, gefolgt von Verben, Adjektiven und teilweise Adverbien. Diese theoretische Annahme kann durch verschiedene Korpora-Untersuchungen bestätigt werden.

Zürn bemerkt aufgrund ihrer Korpusanalyse eine generelle Beschränkung der gefundenen Instanzen auf die vier Wortklassen Substantiv, Verb, Adjektiv und Adverb, während Substantive insgesamt etwa 94\% und Adverbien nur für ein vernachlässigbares Fragment des Korpus ausmachen (0,03\%). Verben und Adjektive liegen bei etwa 3\%. ${ }^{41}$ Yangs Untersuchung eines Korpus aus dem Jahr 1980 zeigt ähnliche Ergebnisse, wobei der Anteil der Substantive bei etwa 93\%, die Verben bei etwa 4\% und die Adjektive bei 2,5\% liegen. Adverbiale Anglizismen sind mit nur $0,1 \%$ ebenfalls vernachlässigbar. ${ }^{42}$ Etwa die gleichen Zahlen gelten für Plümers Forschung: Nomen 93\%, Adjektive $4 \%$, Verben $2 \%$, andere $1 \%{ }^{43}$ Es ist höchst interessant, dass Onyskos Analyse des Spiegel-Korpus aus dem Jahr 2000 leicht unterschiedliche Ergebnisse zeigt: Nomina betragen etwa 86\%, Verben und Adjektive etwa die gleichen Zahlen (etwa 5,5\%) und Adverbien machen nur 1\% der analysierte Daten. ${ }^{44}$ Es scheint, als ob sowohl die Verben als auch die Adjektive zum Nachteil von Substantiven, die wiederum im gegebenen Korpus seltener geworden sind, leicht zugenommen haben.

4. Die Integration von Anglizismen in das deutsche morphosyntaktisches System

${ }^{41}$ Vgl. Zürn, Alexandra: Anglizismen im Deutschen. Karlsruhe: Diss. phil.

Universität

Karlsruhe, 2001, S. 108 .

${ }^{42}$ Vgl.Yang, Wenliang: Anglizismen im Deutschen. Tübingen, May Niemeyer

Verlag.,1990, S. 29 .

${ }^{43}$ Vgl. Plümer, Nicole. : Anglizismus - Purismus - Sprachliche Identität.

Frankfurt am

Main, 2000, S. 142.

${ }^{44}$ Vgl. Onysko, Alexander: Anglicisms in German: Borrowing, Lexical

Productivity, and

Written Codeswitching. Berlin, 2007, S. 131.

Y 10 
Da lexikalische Übertragungen zwischen in Kontakt stehenden Sprachen stattfinden, stellt sich das Problem, wie diese Instanzen in ein bereits etabliertes morpho-syntaktisches System mit anderen Regeln als denen in der Ausgangssprache eingebunden sind. Wie Plümer definiert, kann die Integration von Anglizismen definiert werden als die Einbeziehung und Aufnahme eines fremden Lexems in das Sprachsystem der Empfängersprache. ${ }^{45}$ Für die vorliegende Studie ist $\mathrm{zu}$ beachten, dass nur die Integration von Lehnwörtern untersucht werden soll, wobei natürlich auch verschiedene andere Anpassungsmechanismen für die Anpassung von Strukturmerkmalen möglich sind. ${ }^{46}$

Abhängig von der Intensität des Kontakts können verschiedene Grade der Integration von Lehnwörtern gefunden werden, die von der vollständigen Anpassung reichen - in der das Fremdwort die gleichen Eigenschaften wie der Geburtsbegriff in Bezug auf Rechtschreibung, Aussprache und Flexion trägt und folglich nicht ist aus dem Material, das in der Muttersprache des Sprechers vorherrscht, erkennbar - nur geringe Integration mit Textmarkern wie Anführungszeichen oder Kursivschrift, die die Fremdheit eines Begriffes bedeuten. ${ }^{47}$

- Offensichtlich lässt dieses Paradigma Raum für verschiedene Nuancen der Integration. Wichtige Phasen, die an dieser Stelle erwähnt werden sollten, sind hybride Formen, die aus englischem und deutschem Material bestehen, und Beispiele für Kreativität in der Rezipientensprache, die englische aussehende Wörter (z.B. Flip- Flop) bilden, unabhängig von ihrer möglichen Existenz in Englisch (Pseudo-Anglizismen).

Im Hinblick auf die unterschiedlichen Grade der Anpassung eines Kreditwortes ist es wichtig zu berücksichtigen, dass dieser Prozess im Wort selbst erkennbar ist oder nicht. Die zugrunde liegende Integration könnte immer noch in einer Bedeutungs- oder Funktionseinschränkung stattfinden. Wie Aikhenvald bemerkt: „Eine Form oder ein Muster ist nach ihrer Entlehnung wahrscheinlich von der

\footnotetext{
${ }^{45}$ Vgl. Plümer, Nicole. : Anglizismus - Purismus - Sprachliche Identität. Frankfurt am Main, 2000, S. 189 .

${ }^{46}$ Vgl. Aikhenvald, Alexandra Y. : "Grammars in Contact: A Cross-Linguistic Perspective." , 2007, S. 21 .

${ }^{47}$ Vgl.Plümer: 2000, S. 189 .
}

DOI: 10.12816/0054838 
ursprünglichen Ausgangssprache in Bezug auf ihre formale Anpassung sowie ihre Semantik und Funktion verschieden." 48

Diese Unterschiede gelten auch für das Kontaktszenario zwischen Englisch und Deutsch. Wie Onysko treffend bemerkt, funktionieren englische Wörter häufig als morphologisch einfache Basen, die in die flektierenden und derivativen Paradigmen des Deutschen integriert werden können. ${ }^{49}$ Wiederum bedeutet, dass diese einfachen Basen so modifiziert werden müssen, dass sie dem deutschen morpho-syntaktischen System entsprechen.

Es ist besonders aufregend, den Kurs der Anglizismen in Deutsch von ihrer ersten Integration bis heute zu verfolgen. Natürlich kann keine Aufzeichnung von jedem englischen Begriff, der mindestens einmal verwendet wurde, genommen werden, aber der allgemeine Trend sieht ihre Verwendung im Laufe der Zeit ändern, wobei einige Instanzen als eindringlich und fremd, andere als völlig bedeutungsvoll und unverdächtig in der Empfangssprache wahrgenommen werden.

Ein Grund dafür ist der unterschiedliche Grad der Anpassung oder Nativisierung. Beide Begriffe beziehen sich auf die Anpassung von Rechtschreibung, Aussprache und / oder Morphologie von Lehnwörter an die native Struktur der Empfängersprache. ${ }^{50}$ Es ist jedoch wichtig zu betonen, dass die Zeit, in der ein Kredit in eine Fremdsprache aufgenommen wird, keineswegs das einzige Kriterium für seine Nativisierung ist. Wie Viereck betont, findet sich diese Anpassung auch in neueren Anglizismen des 19. Jahrhunderts, ${ }^{51}$ in der Erwägung, dass einige ältere Anglizismen ihre englische Phonologie und / oder Schreibweise beibehalten haben. Wie Görlach und Busse bemerken, könnte es zu ersten Zögern kommen, wenn englische Lehnwörter zum ersten Mal integriert werden: Deutsch verwendet für Groß- und Kleinschreibung, Nummer (und Geschlecht) in Substantiven, Pronomen, Adjektiven und Artikeln sowie für Person, Zahl, Zeit und Stimmung in

${ }^{48}$ Vgl. Aikhenvald, Alexandra Y. : "Grammars in Contact: A Cross-Linguistic Perspective.", 2007, S. 22 .

${ }^{49}$ Vgl. Onysko, Alexander: Anglicisms in German: Borrowing, Lexical

Productivity, and

Written Codeswitching. Berlin, 2007, S. 49.

${ }^{50}$ Vgl. Fischer, Roswitha: "Studying Anglicisms." Anglicisms in Europe, 2008, S. 9 .

${ }^{51}$ Vgl. Viereck, Wolfgang: "Zur Thematik und Problematik von Anglizismen im

Deutschen.",1980, S.11

Y) $V$ 
Verben. Leihwörter werden normalerweise so integriert, dass sie ohne Einschränkungen in jede syntaktische Umgebung passen. Während der Integrationsphase kann es zu einer unreflektierten Phase oder zu Ungewissheit insbesondere bei der Geschlechtszugehörigkeit kommen. $^{52}$

Abgesehen von den erwähnten Schwierigkeiten stellt sich die Integration eines ausländischen Kredits in eine Sprache, dieser Prozess kann auch Anpassungen und Veränderungen mit sich bringen, die in der Empfängersprache selbst stattfinden. Görlach und Busse beschreiben, dass der Import von Lehnwörtern normalerweise eine Änderung in Rechtschreibung, Aussprache und Morphologie mit sich bringt. $\mathrm{Ob}$ diese Wirkung der aufnehmenden Sprache dauerhafte Merkmale verleiht, hängt von der Anzahl der Darlehen und ihrem Grad der Integration ab, was die Nähe der Kontakte und Einstellungen der betroffenen Sprachgemeinschaft widerspiegelt ${ }^{53}$. Die folgenden Absätze versuchen, die Frage zu beleuchten, inwieweit die Integration von Anglizismen Schwierigkeiten bereitet oder möglicherweise neue, oder vielmehr bereits bestehende Merkmale in der deutschen Sprache stärkt.

\subsection{Nominale Anglizismen}

Wie wir gesehen haben, bilden Substantive die mit Abstand größte Gruppe von Anglizismen im Deutschen, da ihr hoher semantischer Gehalt ihre Integration erleichtert. Aber auch ihre Anpassung an das deutsche morpho-syntaktische System erfordert Änderungen sowohl ihrer eigenen Struktur als auch bis zu einem gewissen Grad der Empfängersprache. Im Folgenden soll ein Überblick über die Kontaktparameter Geschlechterzuordnung, Pluralbildung und Fallflexion als Grundlage für eine Bewertung von Sprachanreicherung und Sprachveränderung dienen. Sie stellen die markantesten Paradigmen hinsichtlich der Integration nominaler Anglizismen in Deutsche dar. ${ }^{54}$

\subsubsection{Geschlechtszuweisung}

Generell lässt sich feststellen, dass die Geschlechtszugehörigkeit zu den Anglizismen den deutschen Konventionen der Geschlechterkonvention folgt, da das Geschlecht dem Deutschen

\footnotetext{
${ }^{52}$ Vgl.Görlach, Manfred and Ulrich Busse: "German." English in Europe., 2007, S.23.

${ }_{53}$ Ebda: S. 23.

${ }^{54}$ vgl. Onysko: 2007, S.151.
}

DOI: 10.12816/0054838 
innewohnt, im Englischen aber nicht vertreten ist. ${ }^{55}$ Es ist nicht möglich, alle Konventionen aufzuzählen, die die Zuordnung von Geschlecht von Fremdwörtern auf Deutsch beeinflussen, aber es sollte beachtet werden, dass dieser Prozess nach allgemein anerkannten morphologischen, phonologischen und semantischen Regeln funktioniert. Carstensen weist darauf hin, dass die Zeit der Kreditaufnahme ebenfalls kein entscheidender Parameter in der Geschlechterverteilung ist, da einige ältere Kredite immer noch zwischen zwei Geschlechtern oszillieren, während einige neuere ein definiertes Geschlecht zugeordnet sind und eine große Stabilität aufweisen. ${ }^{56}$

Infolgedessen wurde das Thema Geschlechterverteilung oft als von Ausnahmen und Eigenheiten durchdrungen betrachtet, die Geschlechtervorhersagen im gesamten deutschen Lexikon unmöglich machen. ${ }^{57}$ In der Tat konnten viele Begriffe ihren anfänglichen Unsicherheitszustand nicht verwerfen. Dies lässt sich im Diskurs über die geschlechtsspezifische Zuschreibung, beispielsweise des Wortes Laptop (männlich oder sächlich) oder E-Mail (weiblich oder sächlich) sehen. $^{58}$

Diese Unsicherheiten sind jedoch nicht nur ein Merkmal der Integration von Anglizismen, sondern auch anderer ausländischer Kredite und Neologismen. Selbst Begriffe, die schon lange im deutschen Lexikon existieren, können von manchen Muttersprachlern zu falschen Geschlechterzuweisungen neigen, wie z. B. in (der / das Pfand), (der / das Vorderteil) oder (der/oder die Krake ), obwohl der Krake in der Standarddeutschen korrekt ist . Diese Verwirrung ist auch im Umgang mit Neologismen und Eigennamen zu spüren, etwa im klassischen Streit um die oder das Nutella.

In der Konsequenz kann davon ausgegangen werden, dass die Probleme, die sich bei der Vergabe eines bestimmten Geschlechts an Kredite ergeben, nicht nur auf Anglizismen beschränkt sind, sondern sich auch in der Fremd- und sogar in der deutschen Etymologie zeigen. Es lässt sich jedoch folgern, dass durch die Einfuhr einer so hohen Anzahl an englischen Begriffen mehr Wörter einem Geschlecht in Deutsch zugeordnet werden müssen, was natürlich zu mehreren Fällen führt, in denen die geschlechtsspezifische Zuordnung kompliziert ist.

${ }^{55}$ Vgl. Onysko: 2007, S.151.

${ }^{56}$ Vgl. Carstensen, Broder: "Das Genus englischer Lehn- und Fremdwörter im Deutschen.", Tübingen, 1980, S. 67-68.

${ }^{57}$ Vgl. Onysko: 2007, S.151.

${ }^{58}$ Ebda: S. 176.

r 9 


\subsubsection{Pluralbeugung}

Im Gegensatz zur Geschlechterverteilung, bei der die Zeit, in der ein Anglizismus in die deutsche Sprache eingetreten ist, keinen Einfluss auf deren Integrationsgrad hat, scheint die Pluralisierung der Anglizismen zwischen älteren und neueren Krediten zu unterscheiden.

Einige der älteren Anglizismen, besonders jene, die während des Mittelalters übertragen wurden, wurden zu einem so frühen Zeitpunkt integriert, dass sie jetzt vollständig angepasst sind (z. B. Boote, Lotsen) ${ }^{59}$ Im Gegensatz dazu hat die Mehrzahl der neueren Kredite den englischen Plural beibehalten, der regelmäßig durch Hinzufügen eines -s zum Wortstamm gebildet wird. Dieses Formationsmuster wird im Allgemeinen nicht als störendes Merkmal auf Deutsch wahrgenommen, da ,-s für deutsche Wörter, die mit einem Vokal enden (Uhu + s ), regelmäßig ist und im Norden häufig als Dialektmerkmal vorkommt (Jungs); Die Übernahme des englischen Musters kann daher als Stärkung einer bestehenden Kategorie interpretiert werden. “. 60

Mit anderen Worten, englische morphologische Regeln reproduzieren einfach ein Paradigma, das schon im Deutschen gefunden werden kann, und das, wie Onysko feststellt, ,wirft die Frage auf, inwieweit die Pluralformen zusammen mit ihren englischen Basen entlehnt sind oder als inhärente Marker des Plural im Deutschen erscheinen. "61

Abweichungen von dieser Regel finden sich jedoch in Ausnahmen wie die Verwendung der Nullflexion bei Wörtern, die aufer (ein Manager, zwei Manager; ein Computer, zwei Computer) enden, auch bei sonst wenig integrierten Formen (ein Teenager, zwei Teenager); seltene Ausnahmen sind Papiere und Poster. ${ }^{62}$ Diese plurale Formation ist zwar nach normativen englischen Normen inakzeptabel, entspricht aber den deutschen morphologischen Regeln (vgl. ein Arbeiter, zwei Arbeiter) und kann daher auch nicht als das deutsche Flexionssystem beeinflussend oder verändernd angesehen werden.

Nur in wenigen Fällen entwickelt das deutsche System für englische Begriffe eigenständige morphologische Merkmale, was sprachliche Kreativität begründen kann. Wie Viereck feststellt: „Zum Beispiel, , der Drops, der bereits Plural auf Englisch ist, nimmt er eine

${ }^{59}$ vgl.Görlach, Manfred and Ulrich Busse: "German." English in Europe... "2007, S. 24.

${ }^{60}$ Ebda: S. 24.

${ }^{61}$ vgl. Onysko: 2007, S.180

${ }^{62}$ vgl. Görlach/ Busse: 2007, S.24.

DOI: 10.12816/0054838 
neue Pluralform auf Deutsch an, die Dropse ${ }^{63}$, ein Zeichen für einen englischen Begriff, der als reifes, nicht analysiertes Ganzes in die deutsche Sprache eingeht und an dem ein regulärer Plural angebracht ist.Folglich verändert die Integration von nominalen Anglizismen hinsichtlich ihrer Pluralbildung das deutsche morpho-syntaktische System nur insofern, als sie ein bereits bestehendes morphologisches Merkmal verstärken (-s Pluralflexion); ansonsten konvergieren pluralisierte nominale Anglizismen mit der deutschen Grammatik.

\subsection{Verbale Anglizismen}

Die verbalen Anglizismen (z.B. to check - checken, to deal - dealen, trainieren, transferien u.a.), die die zweitgrößte (noch im Vergleich zu nominalen Anglizismen sehr kleine) Gruppe von englischen Krediten bilden, werden in einer Vielzahl von syntaktischen Funktionen verwendet und entsprechend beeinflusst. ${ }^{64}$ Vor allem in ihrer infinitiven Markierung und dem besonderen Stand der Partizipien erlaubt uns die Analyse von Verben, weiter zu untersuchen, wie sich die Integration von Anglizismen vom Standarddeutschen lösen kann. Obwohl verbale Anglizismen nur einen kleinen numerischen Einfluss auf das Deutsche haben, bieten ihre syntagmatische Bedeutung (Valenz, Fallzuordnung), ihre paradigmatische Integration in das deutsche Flexionssystem und ihre mögliche Herleitung mit verschiedenen deutschen Präfixen weitere Einblicke in das Strukturelle Verhalten von Anglizismen auf Deutsch. ${ }^{65}$

Wenn es darum geht, die Arten der englischen verbalen Kredite zu analysieren, die auf Deutsch angenommen werden, ist es notwendig, eine klare Hierarchie hinsichtlich ihrer Verwendung zu definieren. Wie Onysko bemerkt: Interessanterweise folgt die Flexionsintegration von verbalen Anglizismen einer Nutzungsneigung von Infinitivformen ( Partizipien Person ) ( Zahl Tempus ) (Imperfekt) Flexion. Verbalanglizismen treten also am häufigsten im Infinitiv auf. Dieser Zusammenhang hängt mit den Hierarchien der Ausleihbarkeit zusammen, da er auf die Tendenz hinweist, dass die Menge an grammatikalischer Information (Markierung) die Wahrscheinlichkeit der Integration von Kontaktmerkmalen in das native System beeinflusst. $^{66}$

\footnotetext{
${ }^{63}$ vgl. Viereck: 1986, S. 116.

${ }^{64}$ vgl. Onysko: 2007, S. 234.

${ }^{65}$ vgl. Onysko: 2007, S. 230.

${ }^{66}$ vgl. Onysko: 2009, S. 63.

YrI
} 
Mit anderen Worten, je einfacher ein Wort in Bezug auf seine grammatischen Merkmale ist, desto leichter wird es in eine andere Sprache übernommen. Für das Kontaktszenario von Englisch und Deutsch bedeutet dies, dass die Infinitivform die mit Abstand häufigste syntaktische Funktion ist, in der englische Wörter auf Deutsch vorkommen.

\subsubsection{Infinitives Markieren}

Die Unterscheidung zwischen älteren und neueren Krediten, die in Pluralflexionen von nominalen Anglizismen gesehen werden, gilt auch für mündliche Kredite. Lexikalische Übertragungen aus dem 19. Jahrhundert wurden mit dem derivativen Morphem -ier übernommen, so dass sie automatisch flektierbar (boykottieren) sind. ${ }^{67}$ Dieser Infinitivmarker findet sich in den letzten Ausleihen nicht mehr, sondern ist dem bloßen Suffix -en gewichen, das im Allgemeinen den Infinitiv der überwiegenden Mehrzahl der verbalen Anglizismen ableitet. ${ }^{68}$ Eine andere ableitende Möglichkeit ist die -n-Suffixierung, die von bestimmten phonologischen Umgebungen ausgelöst wird. Die allgemeine morphologische Regel kann als die Suffixierung des übertragenen Kernelements mit dem deutschen Infinitivmarker zusammengefasst werden, was zu einem ,geliehenen lexikalischen Nukleus + angepassten Flexion führt. “69 Es ist erwähnenswert, dass einige englische verbale Übertragungen für Null-Beugung optieren. Diese fungieren jedoch nicht als isolierte lexikalische Anleihen, sondern sind als Elemente von Kodeschaltern und Phrasenkonstruktionen in den deutschen Diskurs eingebettet, wie zum Beispiel in „Die Show muss weitergehen ${ }^{\text {“ } 70}$. Darüber hinaus gilt die unproblematische Integration nicht nur für die Infinitivmarkierung, sondern auch für andere flexional markierte Kategorien wie Person, Zahl, Tempus und Stimmung, so dass folglich die überwiegende Mehrheit der verbalen Anglizismen zusammenhängt das reguläre (schwache) Flexionsparadigma deutscher Verben. ${ }^{71}$

Diese markierten Infinitive folgen dem schwachen konjugierten Muster und werden von einigen als, weitere unabhängige morphologische Entwicklungen" angesehen, $d$. h. als ein kreativer Prozess der

\footnotetext{
${ }^{67}$ vgl. Görlach, Manfred and Ulrich Busse: "German." English in Europe... "2007, S.

25.

${ }^{68}$ vgl. Onysko: 2007, S. 230.

${ }^{69}$ vgl. Field: 2002, S. 141.

${ }^{70}$ vgl. Onysko: 2007, S.232.

71 Ebda: S.233.
}

DOI: 10.12816/0054838 
Wortbildung in der deutschen Sprache, wie er in ,testen, jobben, checken und getestet, gejobt [sic], gecheckt bzw. mit gelegentlichen Unsicherheiten wie z. B. gefinisht | gefinished ${ }^{172}$. Demnach werden deutsche Verben unabhängig vom englischen morpho-syntaktischen System integriert, indem bereits vorhandene morphologische Merkmale in Deutsch verwendet werden. Dies kann als eine gute Integration ohne weitere Gefährdung des deutschen grammatischen Systems angesehen werden. Wie Onysko belegt, wird die Integration durch die Tatsache unterstrichen, dass auch Anglizismen, die nicht aus dem Englischen stammen, sondern aus einem deutschen Produktivitätsprozess resultieren, dem gegebenen morpho-syntaktischen System entsprechen: Die strukturelle Schlussfolgerung der infinitival Ableitung von verbalen Anglizismen in Deutsch ist, dass verbale Anglizismen sich regelmäßig mit deutschen Endungen abwechseln, unabhängig von ihrer Entstehung als verbale Anleihen oder als sprachinterne Ableitungen von nominellen Anglizismen. ${ }^{73}$

\subsection{Adjektivische und adverbiale Anglizismen}

Obwohl Adjektivkredite nur einen minimalen Prozentsatz der gesamten Anglizismen auf Deutsch ausmachen, sehen sie sich mit mehreren Schwierigkeiten konfrontiert, wenn es um ihre Integration geht. Die wichtigste Frage ist ihre regelmäßige Zustimmung zu ihren Substantiven in Geschlecht, Person und Zahl ${ }^{74}$ in der attributiven Funktion. ,Dies gilt auch für eine Vielzahl von partizipialen Adjektiven, die von gut integrierten verbalen Anglizismen abgeleitet sind, wie bei boomende 'booming', recycelte 'recycled', gepiercte 'pierced', and ausgepowerte'expend its power, exhausted ${ }^{75}$. Die morphologisch komplexe Integration adjektivischer Anglizismen bringt ,Zögern über die Verwendung von Lehnwörtern in attributiver Position (wenn sie flektiert werden müssen) mit sich, folglich bleiben viele Adjektive auf prädikative Funktionen beschränkt (wo sie nicht eingebogen sind)." Wenn man den Integrationsprozess noch einen Schritt weiterführt, kann ,,[m] Orphemensubstitution (z. B. -y ersetzt durch -ig) einen hohen Konsolidierungsgrad anzeigen (man beachte die konkurrierenden Formen trendy und trendig)“. Als zweiter Integrationsindikator können ,Ersatzformen wie in: rosafarbenes Kleid

\footnotetext{
72 vgl. Viereck: 1986, S.116.

73 vgl. Onysko: 2007, S.233.

74 vgl. Onysko: 2009, S.63.

${ }^{75}$ Ebda: S. 63.

r
} 
vs. das Kleid ist rosa (Farben) ${ }^{6}$, das Kleid ist rosa 'gefunden werden ${ }^{76}$. Es ist interessant, hinzuzufügen, dass diese Ersatzformen in der lässigen Sprache zugunsten der Form, die vermieden werden sollte, abgeschafft werden. Dies könnte teilweise auf die ziemlich ausgefeilte Konnotation der längeren Version und die Präferenz in der lässigen Sprache zurückzuführen sein.

Wie man sieht, erleichtern die nicht markierten Adjektivformen aufgrund ihres morphologisch einfacheren Verhaltens die Integration ins Deutsche. Als Ergebnis kann man davon ausgehen, dass die Adoption englischer Adjektive von einer Verwendung in nicht-flektierten Kontexten bis zur regulären Integration in deutsche morphologische Schemata folgt. Somit sind prädikative Adjektive häufiger als attributive. ${ }^{77}$

Als Ergebnis wird eine Divergenz kaum gefunden, da das morphologische Verhalten prädikativer Adjektiv-Anglizismen Regularität nahelegt.

Was die Integration von Adverbial-Anglizismen betrifft, so sind kaum Kollisionen mit dem deutschen morpho-syntaktischen System erkennbar, da sie in deutscher Sprache nicht gekennzeichnet sind: Da in deutschen Adverbien mit nicht-flektierten Adjektiven nichts zu tun hat; kein Kredit mit dem englischen Adverbial Morphem scheint auf Deutsch aufgenommen zu sein. ${ }^{78}$

Zusammenfassend kann man annehmen, dass adverbiale Anglizismen die geringsten Schwierigkeiten darstellen, wenn es um ihre Integration geht. Adjektivische Anglizismen in prädikativer Funktion passen sich ebenfalls leicht an, da im Deutschen keine Flexion benötigt wird. Attributive Adjektive konfrontieren die Sprecher jedoch mit einigen Problemen bei ihrer Anpassung, die z. T. durch Morphemersatz oder vollständige Ersatzformen gelöst werden. Darüber hinaus ist davon auszugehen, dass adjektivische Anglizismen, wie auch verbale Partizipien, durch ihre Integration zwar zu ungewöhnlichen Formen führen können, sich aber nicht modifizierend auf das deutsche morphosyntaktische System auswirken.

\subsection{Pseudoanglizismen}

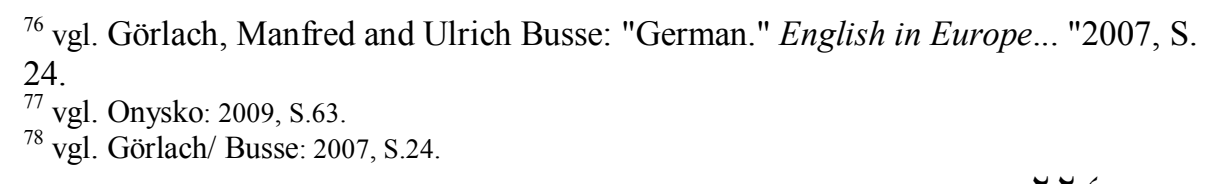

DOI: 10.12816/0054838 
Nicht unbedingt ein Teil des englischen Lexikons, mehrere Instanzen von scheinbar englisch aussehenden und klingenden Wörtern haben Popularität gewonnen. Diese Pseudoanglizismen sind echte Lehnwörter aus dem Englischen, die einen morphologischen Anpassungsprozess an die deutsche Sprache durchlaufen haben. Unter diesen kann nummeriert werden Deutsch Twen von English Twenty, Ger. Pulli aus Engl. Pullover, dt. Happy End aus Engl. Happy End, dt. nicht zuletzt, Ger. kein Eisen von Engl. nichteisen ${ }^{79}$.

\subsubsection{Arten von Pseudoanglizismen}

Wie Onysko feststellt, können englische Formen auch auf Deutsch geprägt werden, ohne dass ein gleichwertiges Modell in englischer Sprache existiert. Dieses Phänomen wird unter dem Pseudoanglizismus zusammengefasst. ${ }^{80}$ Die Bildung von Pseudoanglizismen folgt der Bildung eines auf Analogie basierenden Wortes, das später semantisch uminterpretiert, ersetzt, zusammengesetzt oder verkürzt wird. ${ }^{81}$ Diese Prozesse führen zu einer Verschiebung des Verständnisses oder sogar Unverständnisses für englische Muttersprachler. Es ist wichtig hinzuzufügen, dass Pseudo-Anglizismen nicht unbedingt als geliehene Gegenstände gelten, da ihre Bildung auf englischen Morphemen basiert, aber durch sprachinterne Kreativität auf Deutsch erreicht wird: Aus diesem Grund sollten solche nicht-englischen Prägungen besser als Hinweis auf die Integration der Bestandteile (die dann als Elemente der Empfängersprache produktiv werden können) und nicht als Kreditaufnahme verstanden werden. ${ }^{82}$

Carstensen unterscheidet zwei Arten von Anglizismen, die er definiert :Analoge lexikalische Scheinentlehnungen ${ }^{83}$ und analoge lexikalische Scheinentlehnungen mit zufälligerer englischer Formentsprechung ${ }^{84}$; meine Kursivschrift). Die erste Art, analoge lexikalische Scheindarlehen, ist durch die Prägung eines neuen deutschen Wortes in Analogie $\mathrm{zu}$ einem bereits bestehenden englischen Begriff gekennzeichnet. Es ist wichtig $\mathrm{zu}$ beachten, dass, obwohl englische Morpheme kombiniert werden, diese spezielle Kombination von Morphemen oder ihre Bedeutung im Englischen nicht existiert. Laut Carstensen ist diese Kategorie bei weitem die größte und die Beispiele,

\footnotetext{
${ }^{79}$ vgl. Viereck: 1986, S.116.

${ }^{80}$ vgl. Onysko:2007, S. 217.

${ }^{81}$ Ebda: S.218.

${ }^{82}$ vgl. Görlach/ Busse: 2007, S. 29.

${ }^{83}$ vgl.Carstensen: 1981, S. 176.

${ }^{84}$ Ebda: S.178.

ro
} 
die er gibt, unterstreichen den Prozess der Einbürgerung sehr gut: Unter einigen scheinbar altmodischen Begriffen wie „Longseller" oder „Trendmaker" schlägt er "Showmaster" vor und seine Analogien "Quizmaster“ und „Talkmaster" als typischer Begriff in dieser Kategorie. ${ }^{85}$

Die zweite von Carstensen beschriebene Art von Pseudoanglizismen umfasst Kredite, die in Analogie zu Englisch aufgebaut sind, mit dem Unterschied, dass diese Kombinationen tatsächlich in der englischen Sprache existieren. Interessanterweise haben diese Wörter in beiden Sprachen unterschiedliche Bedeutungen, so dass Carstensen sie als homonyme Homonyme bezeichnen kann. ${ }^{86}$ Diese Ähnlichkeiten verursachen oft Schwierigkeiten im gegenseitigen Verständnis, wie es beim Bedeutungsunterschied des Wortes „Callboy“ für Englisch- und Deutschsprachige der Fall ist, wenn es zum ersten Mal als „Person definiert wird, die Schauspieler aus ihren Umkleidekabinen ruft, wenn es Zeit für sie ist, auf die Bühne zu gehen" und für letztere als männliches Gegenstück zu einem Callgirl. ${ }^{87}$ Um dies in Vierecks adäquaten Worten auszudrücken: Diese „Fälle, in denen der Deutsche seine eigenen semantischen Furchen pflügt, sind in letzter Zeit stark angestiegen [...] Diese Tendenz kann als eine natürliche Phase im Prozess der semantischen Integration angesehen werden ${ }^{688}$

An dieser Stelle wäre angebracht, dass man zu dieser zweiten Kategorie von Pseudoanglizismen das oft gehörte „Handy“ als Bezeichnung für Mobiltelefone in Deutschland hinzufügen kann. Diese Analogie lässt kaum Zweifel hinsichtlich ihrer Etymologie, die die Bequemlichkeit des besagten Geräts betont, aber ihre Wortklasse als ein Adjektiv macht es für einen englischen Sprecher schwierig, seine Bedeutung zu verstehen. Wie Carstensen feststellt, basiert das Verständnis von Begriffen, die zu dieser Kategorie gehören, weitgehend auf Kontexte, während analoge lexikalische Pseudo-Kredite von englischen Muttersprachlern im Allgemeinen leicht $\mathrm{zu}$ verstehen und zu reproduzieren sind. Es ist auch interessant zu bemerken, dass es einen neuen Trend in Deutsch gibt, andere Begriffe für „Handy“ zu verwenden, wie zum Beispiel

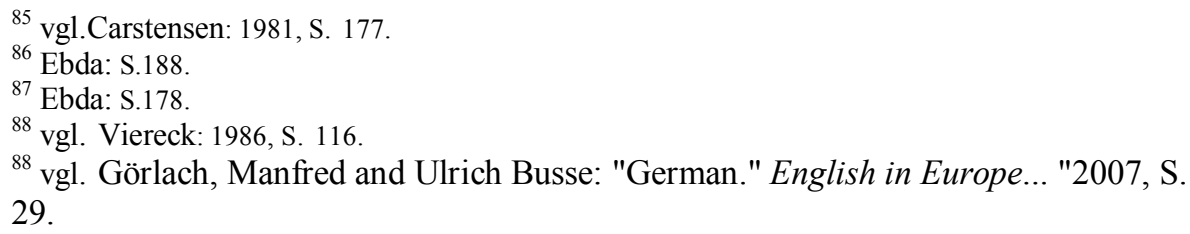

DOI: 10.12816/0054838 
„Mobiltelefon“", eine Lehnübersetzung (Calque) in Analogie zur englischen Quelle „Handy“ Dieser Begriff ist ein neuer, indirekter Anglizismus, der nach und nach das Alte ersetzt, was aufgrund seiner typisch englischen phonologischen Struktur als etwas „eindringlich“ der deutschen Sprache wahrgenommen werden kann, eine Frage, die es in Zukunft zu beantworten gilt.

\subsubsection{Einstellungen zur Verwendung von Pseudoanglizismen}

Der häufige Gebrauch von scheinbar englischen Wörtern scheint das Bedürfnis des Benutzers zu unterstreichen, zumindest Englisch zu hören, obwohl er nicht genug Kompetenz hat, um die Sprache selbst zu beherrschen. Görlach und Busse setzen Ignoranz als einen häufigen Grund für die Bildung von Pseudoanglizismen voraus: „Unvollständige Kompetenz in der Gebersprache (oder ein Nicht-Akzeptieren seiner vorgeschriebenen Normen für geliehene Gegenstände) kann zu englisch aussehenden Gegenständen führen, die es im Englischen selbst nicht gibt. “689 Auch wenn die Einfügung scheinbar englischer Gegenstände möglicherweise nicht zu Schaden kommen soll, verkörpert sie eine sachlich leere Ausdrucksweise und deutet lediglich auf den (fehlgeleiteten) Wunsch des Sprechers an, sachkundig und modern zu erscheinen. ${ }^{90}$ Dass dies gilt, zeigt sich insbesondere im Bereich der Technik, wo innovative deutsche Begriffe wie Beamer oder Handy auf Aktualität und technische Pionierarbeit schließen lassen.

Der schwierige Stand der Pseudoanglizismen ist vor allem in ihrer Bedeutungsleere beim Sprecherkontakt zu sehen. Während die meisten Pseudo-Kredite in der deutschsprachigen Umgebung verständlich sind, wo sie entstanden sind, wird das Verständnis im Kontakt mit englischen Muttersprachlern behindert. Folglich kann die Einordnung eines Wortes als Pseudoanglizismus nur anhand von Nachschlagewerken der englischen Sprache beantwortet werden und ist vom Urteilen von Muttersprachlern abhängig. ${ }^{91}$

Auf der anderen Seite bedeutet dieses gegenseitige Unverständnis nicht unbedingt die „falsche“ Verwendung einer Sprache in einem

${ }^{89}$ Vgl. Onysko: 2009, S.54.

${ }^{90}$ Vgl. Onysko: 2007, S.217. 
präskriptiven Sinn, zumindest was die Rolle der deutschen Produktivität betrifft. Angesichts des zunehmenden globalen Status des Englischen ist es nur natürlich, dass auf der ganzen Welt verschiedene Varietäten entstehen, jede mit ihren eigenen Besonderheiten in Lexik, Morphologie oder Phonologie. Gleiches gilt für deutsche Varietäten, bei denen die Sprecher aufgrund der unterschiedlichen regionalen Variation auf gegenseitige Unverständlichkeit stoßen können. Das einzige Problem bei der Verwendung von Pseudoanglizismen besteht in der angenommenen Internationalität und der Überzeugung des Sprechers, dass phonologische Ähnlichkeiten Verständnis bei Muttersprachlern voraussetzen. Es ist wahrscheinlich, dass die Kommunikation schief geht, wenn es keine gemeinsame Basis des Verstehens gibt, weshalb Pseudo-Anglizismen von vielen verurteilt werden.

Nichtsdestoweniger scheint die Prägung von Pseudo-Anglizismen unvermeidbar zu sein, angesichts der Kreativität, mit der das Deutsche die Regeln des Standardenglisch verbiegt. Aus beschreibender Sicht kann dieser Prozess ,ein deutscher lexikalischer Beitrag zu einer Vielzahl von globalen Englisch“ sein und folglich „Pseudoanglizismen repräsentieren die produktive Verwendung von Englisch in einem überwiegend deutschen sprechenden [sprachkulturellen] Bereich.“92 Als Konsequenz kann eine tolerantere Herangehensweise an die Verwendung von Pseudo-Anglizismen angebracht sein, insbesondere im Hinblick auf die zahlreichen und unterschiedlichen Varianten von Englisch auf der ganzen Welt

\section{Schlussfolgerung}

Das vorliegende Papier hat versucht, die Einflüsse des Englischen auf die deutsche Sprache im Laufe der Zeit zu untersuchen, insbesondere im Hinblick darauf, wie geliehene Elemente in das morpho-syntaktische System des Deutschen integriert werden. Es hat gezeigt, dass die beiden Sprachen immer sprachlich eng verbunden waren; und diese intensive Übertragung von Englisch auf Deutsch ist kein Phänomen der letzten Zeit. Anglizismen wurden immer als eindringlich empfunden, um die Sprache zu verderben. Es gibt jedoch bestimmte Mechanismen, die es ermöglichen, dass englische Begriffe in die deutsche Sprache eingebettet werden, und es kann davon ausgegangen werden, dass der

92

DOI: 10.12816/0054838

rTA 
größere Teil der Anglizismen mit der Regel des deutschen morphosyntaktischen Systems übereinstimmt.

Die Ausnahmen, die dieses Papier gezeigt hat, können in der Beugung des Genitivs gefunden werden und die Flexion von attributiven adjektivischen Anglizismen. Es ist bereits angedeutet worden, dass die Entwicklung der ersteren auf der Tatsache beruhen könnte, dass der Genitiv im Deutschen doppelt ausgeprägt ist und dass die Null-Flexion von Anglizismen Paradigmen verstärken kann, die bereits in der weiblichen Deklination erkennbar sind. Attributive AdjektivAnglizismen scheinen jedoch keine ernsthafte Bedrohung für die deutsche Grammatik zu sein, da ihre Integration tendenziell zu dubiosen Formen führt, aber dennoch nicht gegen bestehende morphologische Regeln verstößt. Direkt geliehene, nicht analysierte Adjektive können als Fälle von Codeswitching angesehen werden, in denen das englische Suffix den Anglizismus als ein Beispiel für eine isolierte, nicht etablierte Kreditaufnahme markieren kann. ${ }^{93}$

Zusammenfassend sind die Fälle, in denen Divergenzen auftreten können, relativ gering im Vergleich $\mathrm{zu}$ Anglizismen, die leicht $\mathrm{zu}$ integrieren sind:

Die Korpus-basierten Verallgemeinerungen unter den zahlenmäßig kleineren Typen von verbalen, adjektivischen, adverbialen und anderen Anglizismen heben die reguläre, nicht systemische Integration von englischen Anleihen ins Deutsche hervor. Morphologisch syntaktisch verhalten sich die Anglizismen im Wesentlichen wie andere deutsche Objekte und neigen dazu, Nutzungskontexte vorzuziehen, die keine Flexionsintegration erfordern. ${ }^{94}$

Zusätzlich unterstützt dieses Papier die Theorie eines Fernkontakts zwischen Englisch und Deutsch. Fischer, der sich auf Busse und Onysko stützt, fasst zusammen, dass die Anglizismen tatsächlich zunehmen, obwohl ihre Wirkung im Vergleich zur Gesamtanzahl der alltäglichen Wörter zahlenmäßig niedrig bleibt. $^{95}$ Dies rekapituliert Onysko folgendermaßen:

Der Einfluss des Englischen auf Deutsch bleibt zahlenmäßig klein und widerspricht nicht den deutschen morpho-syntaktischen Konventionen der Sprache, Obwohl er in den Formen lexikalischer Anleihen und Code-Switches spürbar ist . Die Prävalenz lexikalischer Anleihen und ihre Verteilung auf verschiedene Arten von Sprache spiegelt

\footnotetext{
${ }^{93}$ Vgl. Onysko: 2009, S.70.

${ }^{94}$ Ebda: S.64.

${ }^{95}$ Vgl. Fischer, Roswitha: "Studying Anglicisms." , 2008, S.3.

rYq
} 
Vorhersagen von Kredithierarchien wider und betont, dass die Situation der Fremdsprachenkontakte zwischen Englisch und Deutsch in der Tat sehr niedrig ist. ${ }^{96}$

Was hier verändert worden ist, ist die Einstellung zum Gebrauch von Anglizismen. Die Tatsache, dass Adjektive und Verben in den Korpusbasierten Studien, die verglichen worden sind, zuzunehmen schienen, könnten auf einen Weg der Integration hinweisen, der allmählich seine Strenge verliert. Selbst dubiose Formen werden von der Öffentlichkeit akzeptiert, und unklare Formen existieren nebeneinander. Da die Medien, insbesondere das Internet, die Kontaktbereiche zwischen den Sprachen und auch zwischen den Sprechern derselben Sprache erweitern, ist mehr Raum für verschiedene Sprachen erlaubt, und was früher aus einer präskriptiven Sicht als „falsch“ angesehen wurde, bewegt sich nun zu einem tolerierten Merkmal einer Sprache.

Sprachveränderung kann nicht willkürlich kontrolliert werden, geschweige denn mit Sicherheit vorhergesagt werden, denn der Sprachwandel beruht zu einem großen Teil auf den Einstellungen des Sprechers, die ,die meisten allgemein gültigen Vorhersagen [...] verletzen können und manchmal auch dazu führen [...], kontaktbedingte Veränderungen bleiben im Wesentlichen unberechenbar. ${ }^{\text {(697 }}$ Aus diesem Grund bleibt die Entwicklung des Deutschen unter dem Einfluss der englischen Sprache ein Mythos, und nur Verallgemeinerungen können aus dem vorliegenden Material gezogen werden.

Aus der aktuellen Situation zwischen Englisch und Deutsch ist anzunehmen, dass das Kontaktszenario weiterhin eine große Offenheit mit relativ wenigen dauerhaften Änderungen der deutschen Grammatik aufweisen wird. Die Veränderungen im deutschen morphosyntaktischen System können nur mit dem Englischen in Verbindung gebracht werden, obwohl nicht eindeutig behauptet werden kann, dass diese Veränderungen nicht von selbst geschehen würden, so wie die Entwicklung der eigenen Sprache durch die Deutschen. Das Problem, „wie man beweist, dass kontaktbedingte Veränderung stattgefunden hat - das heißt, wie man zeigt, dass ein bestimmtes Merkmal oder eine Gruppe von Merkmalen in einer bestimmten Sprache aufgrund von Sprachkontakt vorhanden ist. “98 wird in Zukunft ein Problem bleiben

\footnotetext{
${ }^{96}$ Vgl. Onysko: 2009, S.71.

${ }^{97}$ Vgl. Thomason: 2001, S.61.

${ }^{98}$ Vgl. Thomason, Sarah G. : Language Contact. Edinburgh: Edinburgh University Press, 2001, S.61.

DOI: $10.12816 / 0054838$ 
insbesondere im Hinblick auf die zunehmende Verschränkung zwischen Sprachen. Als vorübergehende Schlussfolgerung kann festgehalten werden, dass, obwohl Anglizismen im Deutschen nicht immer notwendig und oft unpassend oder irritierend sind, sie immer ein Grundbestandteil unserer Sprache bleiben und waren. Die Möglichkeit der Lehnwörter, eine Sprache zu bereichern, erweist sich als ihr stärkster Vorteil, und durch die Sprache wurden Kontaktsprachen weltweit mit Ausdrücken für unbekannte Konzepte und Bedeutungsnuancen ausgestattet.

\section{Literaturverzeichnis}

Aikhenvald, Alexandra Y. 2007. "Grammars in Contact: A Cross-Linguistic

Perspective." Grammars in Contact: A Cross-Linguistic Typology. Aikhenvald, Alexandra Y. and Robert M.W. Dixon (eds.). Oxford: Oxford University Press. 1-66.

Broder Carstensen, Ulrich Busse. 2001. Anglizismen-Wörterbuch: der Einfluss des Englischen auf den deutschen Wortschatz nach 1945. Band 1 (A-E), Berlin/New York 2001, S. 61*., ISBN 3-11012854-3. 
Carstensen, Broder. 1981. "Lexikalische Scheinentlehnungen des Deutschen aus dem Englischen." Kontrastive Linguistik und Übersetzungswissenschaft. Kühlwein, Wolfgang, Gisela Thome and Wolfram Wilss (eds.). München: Fink. 175-182.

Carstensen, Broder. 1980. "Das Genus englischer Lehn- und Fremdwörter im

Deutschen." Studien zum Einfluß der englischen Sprache auf das Deutsche/Studies on the Influence of the English Language on German. Viereck, Wolfgang (ed.), Tübingen: Narr. 37-75.

Clyne, Michael. 2003. Dynamics of Language Contact: English and Immigrant Languages. Cambridge: Cambridge University Press.

Coetsem, Frans Van. 2000. A General and Unified Theory of the Transmission Process in Language Contact. Heidelberg: Winter.

Dieter Herberg/Michael Kinne/Doris Steffens. 2004. Neuer Wortschatz: Neologismen der 90er Jahre im Deutschen, Berlin/New York .

Duden.2001. Das Herkunftswörterbuch. Bibliographisches Institut, Mannheim/Leipzig/Wien/Zürich . ISBN 3-411-04073-4.

Field, Fredric W. 2002. Linguistic Borrowing in Bilingual Contexts. Amsterdam, Philadelphia: John Benjamins.

Fischer, Roswitha. 2008. "Studying Anglicisms." Anglicisms in Europe: Linguistic Diversity in a Global Context. Fischer, Roswitha and Hanna Pułaczewska (eds.). Newcastle upon Tyne: Cambride Scholars Publishing. 1-14.

Görlach, Manfred. 2007. "Introduction." English in Europe.

Görlach, Manfred (ed.), Oxford, New York: Oxford University Press. 1-12.

Görlach, Manfred. 2003. English Words Abroad. Amsterdam, Philadelphia: John

Benjamins.

Görlach, Manfred and Ulrich Busse. 2007. "German." English in Europe. Görlach, Manfred (ed.), Oxford, New York: Oxford University Press. 13-36.

Junker, Gerhard H. 2003. Der Zeitgeist spricht Englisch. in: Zabel, 
مجلة الدراسات التربوية والانسانية ـ كلية التربية ـ جامعة دمنهور ـ المجلد الحادى عشر - العدد الثانى - لسنة 9 ب ـr

Hermann (Ed.). Denglisch, nein danke! Zur inflationären

Verwendung von Anglizismen und Amerikanismen in der deutschen Gegenwartssprache. Paderborn: IFB Verlag, 119

Muhr, Rudolf. 2004. Anglizismen als Problem der Linguistik und Sprachpflege in Österreich und Deutschland zu Beginn des 21. Jahrhunderts. in: Muhr, Rudolf; Kettmann, Bernhard (Hrsg.). Eurospeak - Der Einfluss des Englischen auf europäische Sprachen zur Jahrtausendwende. Frankfurt am Main: Peter Lang GmbH, 11

Onysko, Alexander. 2009. "Divergence with a Cause? The Systematic Integration of Anglicisms in German as an Indication of the Intensity of Language Contact." Englischer Sprachkontakt in den Varietäten des Deutschen. Pfalzgraf, Falco (ed.), Wien, Frankfurt a. M.: Peter Lang. 53-74.

Onysko, Alexander. 2007. Anglicisms in German: Borrowing, Lexical Productivity, and Written Codeswitching. Berlin: de Gruyter.

Peter Eisenberg: Anglizismen im Deutschen. In: Deutsche Akademie für Sprache und Dichtung, Union der deutschen Akademien der Wissenschaften (Hrsg.): Reichtum und Armut der deutschen Sprache. Erster Bericht zur Lage der deutschen Sprache. De Gruyter, Berlin / Boston, MA 2013, Seiten 57-119. ISBN 978-3-11-033462$\underline{3}$.

Peter Schlobinsk. i2000. Anglizismen im Internet. in: Networx, Nr. 14, Online (PDF; kostenlos, 28 Seiten)

Plümer, Nicole. 2000. Anglizismus - Purismus - Sprachliche Identität. Frankfurt am Main: Peter Lang.

Richard Glahn. 2002. Der Einfluss des Englischen auf gesprochene deutsche Gegenwartssprache. Eine Analyse öffentlich gesprochener Sprache am Beispiel von „Fernsehdeutsch“. 2., durchgesehene Aufl., Peter Lang, Frankfurt am Main, ISBN 3-631-38955-8.

Rudolf Muhr. 2011. Anglizismus. In: $\underline{\text { Gert }}$ Ueding (Hrsg.): Historisches Wörterbuch der Rhetorik. WBG, Darmstadt 1992ff, Band 10, Sp. 37-45.

Stephanie Bohmann.1996. Englische Elemente im rur 
Gegenwartsdeutsch der Werbebranche. Tectum Verlag, ISBN 978-389608-964-9.

Thomason, Sarah G. 2001. Language Contact. Edinburgh: Edinburgh University Press.

Viereck, Wolfgang. 1986. "The influence of English on German in the past and in the

Federal Republic of Germany." English in Contact with Other Languages. Viereck, Wolfgang and Wolf-Dietrich Bald (eds.). Budapest: Akadémiai Kiadó. 107-128.

Viereck, Wolfgang. 1980. "Zur Thematik und Problematik von Anglizismen im

Deutschen." Studien zum Einfluß der englischen Sprache auf das

Deutsche/Studies on

the Influence of the English Language on German. Viereck, Wolfgang (ed.), Tübingen:

Narr. 9-24.

Weinreich, Uriel. 1953. Languages in Contact. Fifth Printing 1967. The Hague, Paris:

Mouton.

Winter-Froemel, Esme. 2008. "Unpleasant, Unnecessary,

Unintelligible? Cognitive and

Communicative Criteria for Evaluating Borrowings and Alternative Strategies."

Anglicisms in Europe: Linguistic Diversity in a Global Context. Fischer, Roswitha and Hanna Pułaczewska (eds.). Newcastle Upon Tyne: Cambridge Scholars Publishing. 16- 41.

Yang, Wenliang. 1990. Anglizismen im Deutschen. Tübingen: May Niemeyer Verlag.

Zürn, Alexandra. 2001. Anglizismen im Deutschen. Karlsruhe: Diss. phil. Universität Karlsruhe. 
مجلة الدراسات التربوية والانسانية ـ كلية التربية ـ جامعة دمنهور ـ المجلد الحادى عشر - العدد الثانى - لسنة 9 . ب r

\section{Internetquellen}

https://www.wikiwand.com/de/Anglizismus

https://alnigo.de/sprachwi/anglizismen.html

Wiktionary: Anglizismus - Bedeutungserklärungen, Wortherkunft, Synonyme, Übersetzungen

Wiktionary: Verzeichnis von Anglizismen - Bedeutungserklärungen, Wortherkunft, Synonyme, Übersetzungen

Wikibooks: Fruchtbringendes Wörterbuch - Lern- und Lehrmaterialien

Annett Stein im General-Anzeiger (Bonn) vom 19. Dezember 2015, Journal Seite 6

Deutsche sprechen schlecht Englisch: Studie der GfK, Die Zeit, 18. Juni 2013

Studie der Gesellschaft für deutsche Sprache, Grafiken (Memento vom 21. Juli 2014 im Internet Archive)

Sprachforscher Göttert: "Das Wort Blockbuster finde ich geschmacklos", Spiegel Online vom 21. November 2013 
ملي سياق البحثل

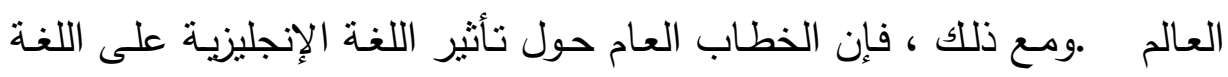

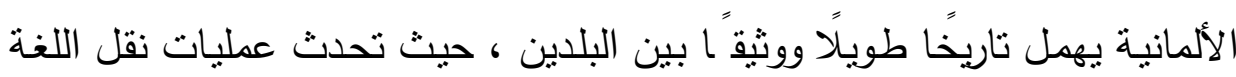

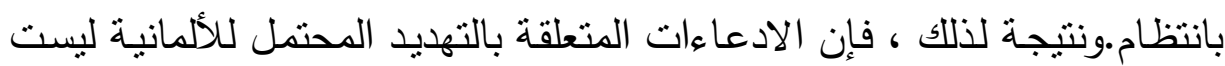

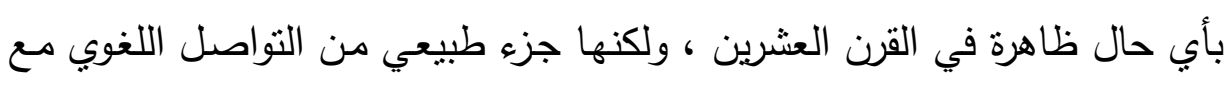

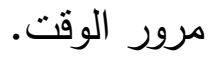

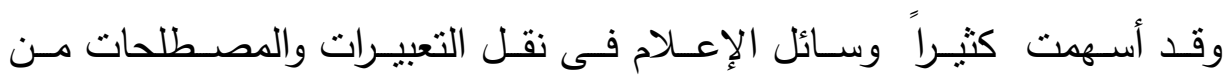

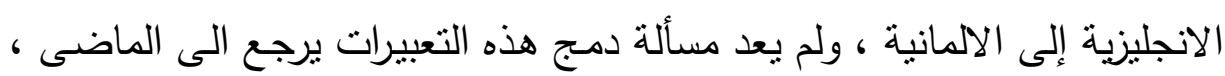

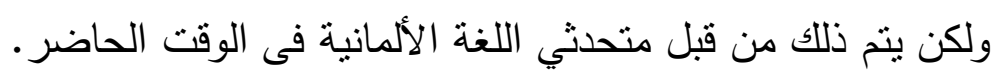

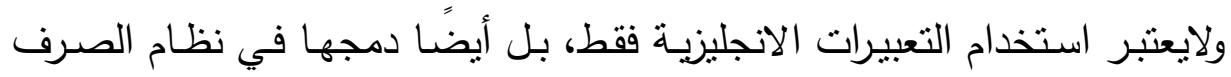

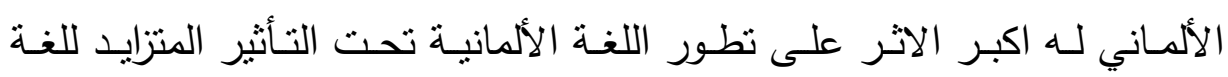
الإنجليزية.

مـع وضـع ذلـك في الاعتبـار ، سـأحاول وصـف حالــة الاتصـال بـين اللغتين

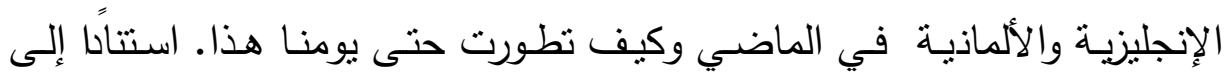

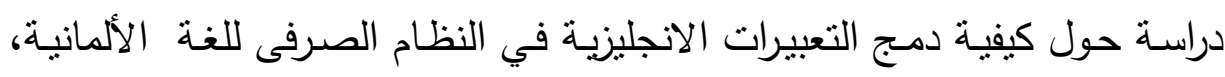
آمل أن أكون قادرا على عمل تتبؤات حول التطور المستقبلي لسيناريو الاتصسال وتأثيره على اللغة الألمانية. يرجى ملاحظة أن هذا البحث يتجاهل حقيقة نقل الكلمات والمصطلحات الألماندانية إلى اللغة الإتجليزية ـ ومن ثم فان هذا البحث يركز بشكل خاص على على التحويلات المعجمية محاولة فهم التغييرات التي تجلبها إلى اللغة الألمانية 